Check for updates

Cite this: RSC Adv., 2019, 9, 26668

\title{
Coordinating gallium hexacyanocobaltate: Prussian blue-based nanomaterial for Li-ion storage
}

\author{
Kaiqiang Zhang, (D) ab Tae Hyung Lee, ${ }^{a}$ Bailey Bubach, ${ }^{c}$ Mehdi Ostadhassan, (D) *c \\ Ho Won Jang, (D) *a Ji-Won Choi (D) ${ }^{* b}$ and Mohammadreza Shokouhimehr (D)*ac
}

Prussian blue analogs (PBAs) are a type of metal-organic framework and have drawn significant attention recently. To date, most are constructed with divalent transition metal ions coordinated to the $\mathrm{N}$ end of a cyanide bridge. In this report, we studied a trivalent gallium ion-based Ga hexacyanocobaltate (GaHCCo), which depicted a face-centered cubic crystal structure. In addition, the synthesized GaHCCo was demonstrated as a cathode material of lithium-ion batteries (LIBS) and was found to exhibit longterm stability, having a capacity retention of $75 \%$ after 3000 cycles of repeated charge-discharge cycling and an extremely high coulombic efficiency of $98 \%$, which was achieved because of a solid-state diffusion controlled Li-ion storage process. After ex situ XRD analysis on the different charge stages, the $\mathrm{Li}$-ion storage in the GaHCCo was attributed to the Co species via the formation of a Li/Co compound. This work will pave the way toward the study of PBAs constructed with trivalent metal ions and provide more insights into the development of high-performance LIBs in the future.

Received 18th May 2019

Accepted 20th August 2019

DOI: $10.1039 / c 9 r a 03746 b$

rsc.li/rsc-advances catalysts, gas sensors, and solid electrolytes and have shown great potential. ${ }^{12-16}$ With regard to the use of MOFs as cathode materials for LIBs, two different methods (direct use or as sacrificial precursors) are typically employed..$^{17,18}$ In particular, Prussian blue analogs (PBAs), which are typical MOFs, are considered one of the most attractive candidates because of their easy of synthesis in aqueous solution at room temperature without the need of additives or post-processing. ${ }^{19}$ Recently, the study of PBAs as cathode materials for LIBs has mainly focused on functionalized PBAs (for example, those treated by etching, oxidation, or combination with other materials such as carbon nanotubes), ${ }^{20,21}$ which results in highly improved electrochemical properties. However, this extra processing increases the fabrication costs during a massive fabrication. Thus, the further study of naked PBAs is logical. With regard to the study of naked PBAs, research groups have mainly focused on $\mathrm{A}_{3}$ $\left[\mathrm{M}^{\mathrm{III}}(\mathrm{CN})_{6}\right]_{2}$ and $\mathrm{A}_{2} \mathrm{M}^{\mathrm{II}}(\mathrm{CN})_{6}$ systems having $\mathrm{A}^{2+}$ ions and $\mathrm{B}_{4}\left[\mathrm{M}^{\mathrm{II}}(\mathrm{CN})_{6}\right]_{3}$ and $\mathrm{BM}^{\mathrm{III}}(\mathrm{CN})_{6}$ systems having $\mathrm{B}^{3+}$ ions $(\mathrm{M}$ : transition metal ions coordinated to $\mathrm{C}$; $\mathrm{A}$ and $\mathrm{B}$ : transition metal ions coordinated to $\mathrm{N}$ ). However, the latter case (i.e., those constructed with $\mathrm{B}^{3+}$ ions) is limited to $\mathrm{Fe}^{3+}$ such as $\mathrm{Fe}^{3+} \mathrm{Fe}(\mathrm{CN})_{6}, \quad \mathrm{Fe}^{3+} \mathrm{Co}(\mathrm{CN})_{6}$, and $\mathrm{Fe}^{3+} \mathrm{Cr}(\mathrm{CN})_{6} \cdot{ }^{22,23}$ Therefore, studies of PBAs constructed with other types of N-coordinated trivalent metal ions are necessary to provide an in-depth understanding for this overlooked issue. To obtain a trivalent metal ion that is soluble and stable in an aqueous solution, we systematically studied the elements in the periodic table. The elements of the boron group in the periodic table have typical trivalent characteristics. We, thus, fabricated the six-fold 
coordinated PBAs using the lighter Ga species. In particular, we synthesized the Ga hexacyanocobaltate (GaHCCo) and further studied its electrochemical properties as a cathode material of LIBs, revealing a solid-state diffusion-controlled Li-ion storage mechanism by utilizing the Co species.

\section{Experimental}

\section{Materials}

GaHCCo nanoparticles (NPs) were synthesized by a coprecipitation method involving the simultaneous dropwise addition of $100 \mathrm{~mL} \mathrm{Ga}\left(\mathrm{NO}_{3}\right)_{3}(0.01 \mathrm{M})$ (Sigma-Aldrich) and $100 \mathrm{~mL} \mathrm{~K}_{3}\left[\mathrm{Co}(\mathrm{CN})_{6}\right](0.01 \mathrm{M})$ (Sigma-Aldrich) to $200 \mathrm{~mL}$ deionized $\mathrm{H}_{2} \mathrm{O}$. The entire synthesis process was carried out at $80{ }^{\circ} \mathrm{C}$ with vigorous stirring. The formation of a precipitate was observed after a period of heating. After a cooling of the mixture to room temperature, the precipitate was separated and rinsed with large amounts of deionized water several times to remove the impurity ions (such as $\mathrm{K}^{+}$) and subsequently dried in a vacuum oven at $60{ }^{\circ} \mathrm{C}$; it was then ready for subsequent use.

\section{Physical characterization}

$\mathrm{X}$-ray diffraction (XRD, D8 Advance, Bruker, $\mathrm{Cu} \mathrm{K} \alpha$ radiation at a fixed incident angle of $2^{\circ}$ ), X-ray photoelectron spectroscopy (XPS, PHI 5000 VersaProbe with an $\mathrm{Al} \mathrm{K}$ source (Sigma Probe, VG Scientific)), Raman spectroscopy (inVia Raman Microscope), and Fourier transform infrared (FTIR, Nicolet iS50, Thermo Fisher) spectroscopy measurements were carried out. The morphology and composition of the samples were investigated using field emission-scanning electron microscopy (FE-SEM, SUPRA 55VP, Carl Zeiss AG), transmission electron microscopy (TEM, JEOL JEM-2100F), energy-dispersive X-ray spectroscopy (EDX). The analysis for a composition of the as-prepared GaHCCo was also carried out using thermogravimetric analysis (TGA) which was performed under $\mathrm{N}_{2}$ flow from room temperature to $700{ }^{\circ} \mathrm{C}$ with a temperature ramp of $10{ }^{\circ} \mathrm{C} \mathrm{min}{ }^{-1}$, as well as X-ray fluorescence measurements (XRF, ZSX-PRIMUS, Rigaku). Quantitative measurements were achieved by using inductively coupled plasma (ICP) for Ga, Co, and $\mathrm{K}$ elements and $\mathrm{EA}(\mathrm{CHNS})$ analysis for $\mathrm{C}, \mathrm{H}$, and $\mathrm{N}$ elements.

\section{Electrode preparation}

A slurry containing GaHCCo, carbon black (Super P Li), and poly(vinylidene)difluoride in a mass ratio of $7: 2: 1$ was prepared by manually grinding the mixed powders and subsequently dispersing them in $N$-methyl-2-pyrolidinone (NMP) in a manner similar to the standard slurry preparation method widely employed in the LIB research communities. In addition, before the injection of NMP, the mixed powders were dried overnight in a vacuum oven at $60^{\circ} \mathrm{C}$. The mixed powder weight was measured before and after vacuum drying to ensure that water was eliminated as much as possible. A working electrode with a mass loading of approximately $3 \mathrm{mg} \mathrm{cm}{ }^{-2}$ was prepared by spreading the slurry on a graphite paper current collector (Alfa Aesar), followed by drying overnight in a vacuum oven at $60{ }^{\circ} \mathrm{C}$.

\section{Electrochemical characterization}

To perform electrochemical measurements, a two-electrode setup was used: the GaHCCo active material was used as the working electrode and sufficient lithium metal to ensure that the capacity was limited solely by the mass of active materials was used as the anode. The electrodes were placed in $1.0 \mathrm{M}$ $\mathrm{LiPF}_{6}$ in a $1: 1$ mixture (by volume) of ethylene carbonate and diethylene carbonate in an argon-filled glove box.

The electrochemical impedance spectrum (EIS) of GaHCCo was measured using an Im6ex ZAHNER instrument in the assembled half-cell. The frequency range used was from $10 \mathrm{mHz}$ to $1 \mathrm{MHz}$, at a voltage amplitude of $10 \mathrm{mV}$.

Cyclic voltammetry (CV) measurements were performed on an electrochemical workstation (WBCS3000, WonATech Co., Ltd., Korea) in the potential range of $2.2-4.5 \mathrm{~V} v s . \mathrm{Li}^{+} / \mathrm{Li}$ at a scan rate of $0.5 \mathrm{mV} \mathrm{s}^{-1}$. Galvanostatic charge/discharge cycling measurements were performed between 2.2 and $4.5 \mathrm{Vvs.} \mathrm{Li}^{+} / \mathrm{Li}$ at various current densities corresponding to 100, 200, 400, 600, 800, and $1000 \mathrm{~mA} \mathrm{~g}^{-1}$. Unless otherwise specified, all the current densities and specific capacities in the present study were calculated based on the weight of the active material, GaHCCo.

\section{Results and discussion}

The proposed crystal structure is shown in Fig. 1; the structure consists of a face-centered cubic crystal structure containing cyanide bridges alternately bonded with $\mathrm{Co}^{3+}$ and $\mathrm{Ga}^{3+}$ centers, having eight cavities (sub-units) within each unit with a size as small as ca. $1 \mathrm{~nm}$. Morphologies of the GaHCCo particles are observed using FE-SEM, and the NPs are found with a size of $c a$. $500 \mathrm{~nm}$ (Fig. 1b). Furthermore, the comprising elements were uniformly distributed throughout the NPs (Fig. 1c). The further magnification images of the particles are obtained using TEM (Fig. 1d) displaying the particle with a diameter of $c a .500 \mathrm{~nm}$ and uniformly distributed elements as shown in the elemental mapping. To investigate this further, TGA experiments were conducted (Fig. 1e), and it revealed a constant decrease in a weight of the product upon heating, confirming the structural weakness of GaHCCo, which is unlike that of other PBAs being able to withstand a temperature up to $400{ }^{\circ} \mathrm{C} .{ }^{24}$ The crystalized feature is depicted in the XRD result. Furthermore, the crystal structure is further confirmed by the Rietveld refinement showing well matched XRD curves for experimentally obtained result and calculated one. The refinement parameters are given in Table 1 demonstrating a space group 225 and lattice parameters of $a=b=c=10.0971 \AA$. The characteristic lattice diffraction peaks in the XRD patterns (Fig. 1f) indicate a typical face-centered cubic crystal structure for the GaHCCo. The characteristic Bragg diffraction peaks (PDF card no. 01-0721431) are well indexed. Here, the standard diffraction peaks are referred to the $\mathrm{PBA} \mathrm{Co} 3\left[\mathrm{Co}(\mathrm{CN})_{6}\right]_{2}$ with a cubic crystal structure due to the absence of the corresponding standard card for GaHCCo. The presence of cyanide bridges was inferred from FTIR (Fig. 1g), which showed characteristic bands at ca. $2200 \mathrm{~cm}^{-1}$. Furthermore, the cyanide bridges were further detected using Raman spectroscopy (Fig. 1h), revealing 
(a)

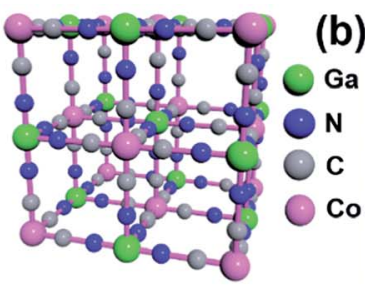

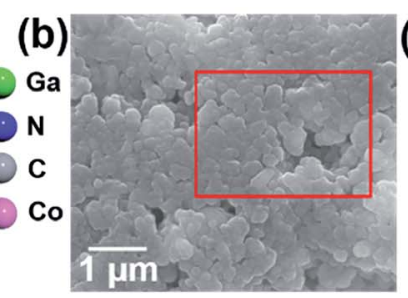
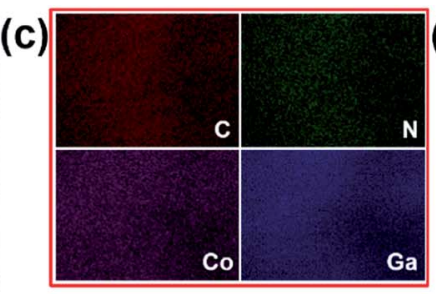

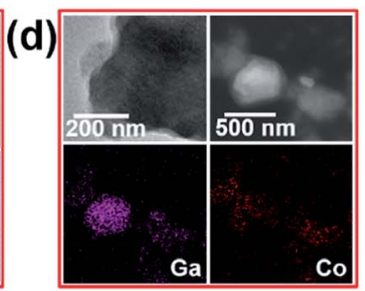

(h)

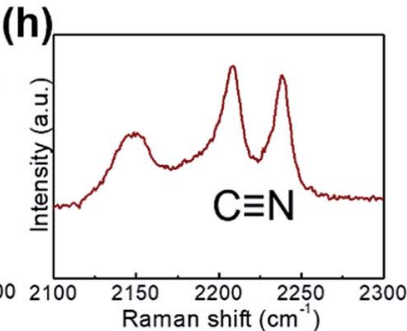

Fig. 1 (a) Crystal structure (b) FE-SEM image, (c) EDX mapping, (d) TEM images, (e) TGA curve, (f) XRD and Rietveld refinement curves, (g) Raman spectrum, and (h) FT-IR spectrum of the GaHCCo.

Table 1 Details of Rietveld refinement parameters for the GaHCCo

\begin{tabular}{ll}
\hline Parameters & Values \\
\hline Space group & 225 \\
$a(\AA)=b=c$ & 10.0971 \\
$V\left(\AA^{3}\right)$ & 1029.4 \\
$\mathrm{C}(x, y, z)$ & $(0.303,0.000,0.000)$ \\
$\mathrm{N}(x, y, z)$ & $(0.1928,0.000,0.000)$ \\
$\mathrm{Ga}(x, y, z)$ & $(0.500,0.500,0.500)$ \\
$\mathrm{Co}(x, y, z)$ & $(0.000,0.000,0.000)$ \\
$R_{\mathrm{Bragg}}$ & 3.607 \\
$R_{\mathrm{wp}}$ & 16.42 \\
$R_{\text {exp }}$ & 10.41 \\
$R_{\mathrm{p}}$ & 13.17 \\
$\mathrm{GOF}$ & 1.58
\end{tabular}

characteristic bands at $c a .2210$ and $2240 \mathrm{~cm}^{-1}$, consistent with reports concerning other PBAs. ${ }^{25}$

The comprising elements are also clearly confirmed in XRF spectra (Fig. 2). The composition elements are screened out at different energies as shown by peaks with high intensities for each element. A quantitative elemental analysis for the GaHCCo by ICP and EA(CHNS) (Table 2) demonstrates the molecular formula $\mathrm{K}_{0.017} \mathrm{Ga}_{0.99} \mathrm{Co}(\mathrm{CN})_{6} \cdot 0.32 \mathrm{H}_{2} \mathrm{O}$. Furthermore, the surface chemical properties of the GaHCCo product are studied using XPS. The wide-survey spectrum (Fig. 3a) indicates bonding nature in GaHCCo. The $\mathrm{C} 1 \mathrm{~s}$ and $\mathrm{N}$ 1s peaks in the deconvoluted spectra are consistent with the presence of cyanide bridges, as indicated by the FTIR and Raman spectra (Fig. $1 \mathrm{~g}$ and $\mathrm{h}$ ). Concomitant $\mathrm{Co}^{3+}$ and $\mathrm{Co}^{2+}$ ions in the deconvoluted Co $2 \mathrm{p}$ spectra with different energies are consistent with the split Raman peaks. Unlike the Co 2p peak, the deconvoluted Ga 2p peak indicates pure $\mathrm{Ga}^{3+}$ after the aqueous synthesis process. Thus, the XPS results demonstrate the bonding state of the formed GaHCCo.

Electrochemical properties of GaHCCo as a cathode material are studied using $\mathrm{CV}$ measurements to determine the electrochemical activity toward Li-ion storage. A stable potential window of 2.2-4.5 V vs. $\mathrm{Li}^{+} / \mathrm{Li}$ without obvious distortion was observed in the CV profile (Fig. 4a). The quasi-rectangular CV curves indicate a predominant double-layer capacitive charge storage feature. The rate performance of GaHCCo is determined at current densities of $100,200,400,600,800$, and $1000 \mathrm{~mA} \mathrm{~g}^{-1}$, with a capacity retention of $42.2 \%$ at $1000 \mathrm{~mA} \mathrm{~g}^{-1}$. The corresponding charge/discharge potential profiles exhibit quasiplateaus at $c a .4 .0 \mathrm{~V} v$ s. $\mathrm{Li}^{+} / \mathrm{Li}$ (Fig. $4 \mathrm{c}$ ). A capacity differential (dQ/dV) (Fig. 4d) exhibits a pair of depressed humps as marked in the differential curves suggesting the charge/discharge plateaus in the voltage profiles (Fig. 4c). The long-term repeated charge/discharge process is shown in Fig. 4e, where an initial capacity of $c a .80 \mathrm{~mA} \mathrm{~h} \mathrm{~g}^{-1}$ after the activation phase is obtained. A high capacity retention of $75 \%$ is achieved after 3000 cycles of successive charge/discharge testing, corresponding to an average loss in capacity of $0.008 \%$ for each cycle, although some fluctuation in the electrochemical capacities is observed. Notably, a high coulombic efficiency of $c a .98 \%$ is achieved during the long-term cycling measurements (Fig. 4e). The voltage profiles during the long-term capacity test are shown in Fig. $4 \mathrm{f}$, revealing the tedious charge/discharge process with quasi-plateaus at around $3.9 \mathrm{~V} v s . \mathrm{Li}^{+} / \mathrm{Li}$ (charge) and $3.3 \mathrm{~V}$ vs. $\mathrm{Li}^{+} / \mathrm{Li}$ (discharge) for each cycle, which can be described as Li-ion redox reactions with local equilibrium. ${ }^{26,27}$

The Li-ion storage process of GaHCCo is further studied using the ex situ XRD method (Fig. 5). The XRD spectra of the samples charged to $2.2,3.0$, and $3.6 \mathrm{~V} v s$. $\mathrm{Li}^{+} / \mathrm{Li}$ reveal the collapse of the GaHCCo structure, which is similar with the previously reported $\mathrm{Co}_{3}\left[\mathrm{Co}(\mathrm{CN})_{6}\right]_{2}$ as anode for potassium-ion batteries. ${ }^{28}$ This can be further observed in the sample after a repeated charge/discharge cycling test (Fig. 6b), where the characteristic peaks of GaHCCo are greatly eliminated. A newly formed peak (corresponding to $\mathrm{Li}_{0.73} \mathrm{CoO}_{2}$ ) at $c a .18^{\circ}$ in the XRD pattern (Fig. 5d) indicates that the corresponding charge/ discharge process is mainly achieved by bonding with Co 

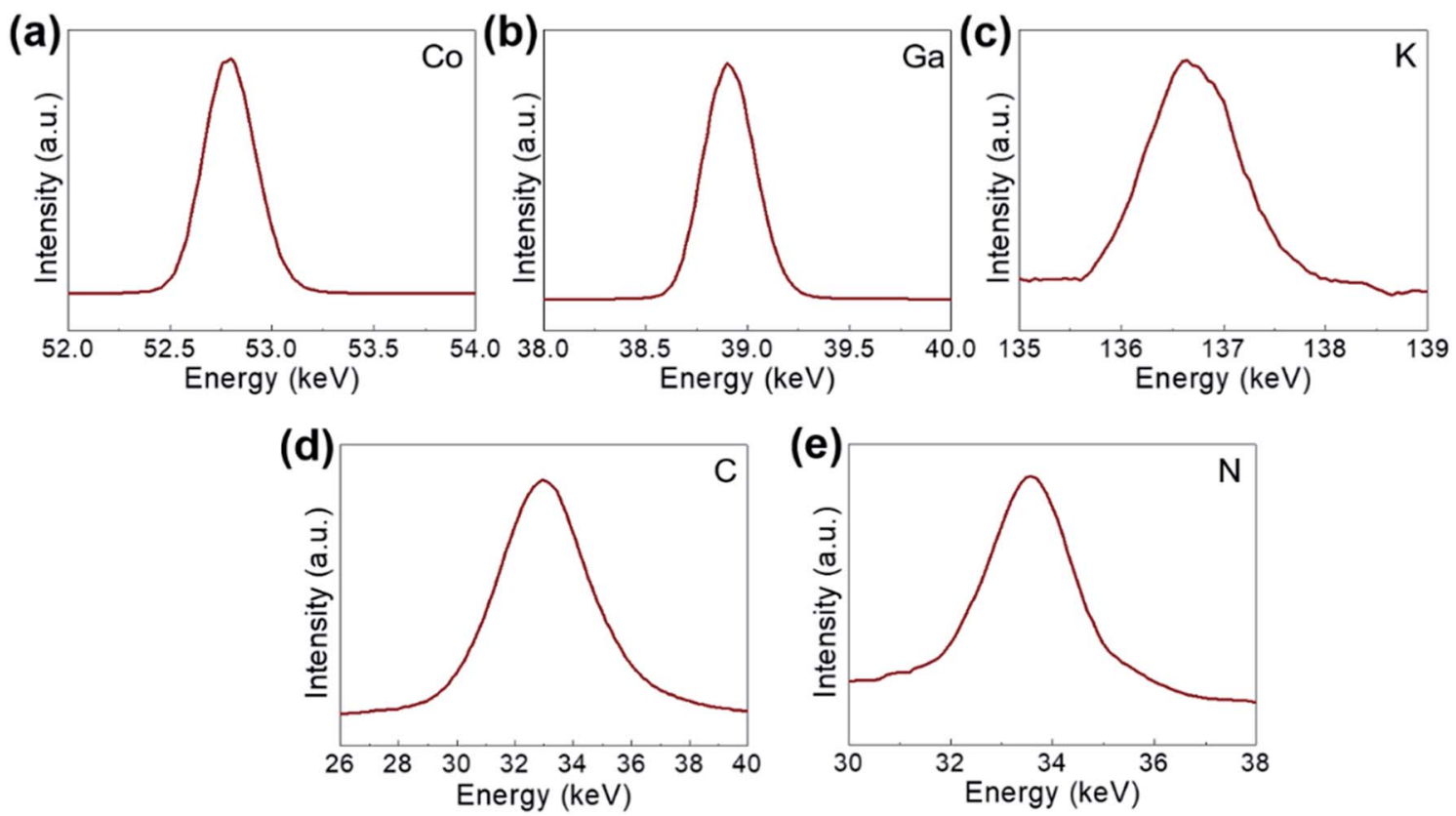

Fig. 2 XPS spectra of the synthesized GaHCCo: (a) Co 2p, (b) Ga 2p, (c) K 2p, (d) C 1s, and (e) N 1s.

Table 2 EA(CHNS) for $\mathrm{C}, \mathrm{H}, \mathrm{N}$ elements and ICP for $\mathrm{Co}, \mathrm{Ga}$, and $\mathrm{K}$ elements measurements of the as-prepared GaHCCo

Element $\quad \mathrm{C}(\mathrm{wt} \%) \quad \mathrm{H}(\mathrm{wt} \%) \quad \mathrm{N}(\mathrm{wt} \%) \quad \mathrm{Co}(\mathrm{wt} \%) \quad \mathrm{Ga}(\mathrm{wt} \%) \quad \mathrm{K}(\mathrm{wt} \%)$

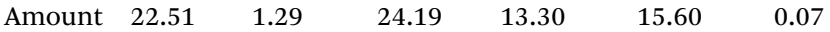

species instead of Ga. On increasing the cutoff voltage, the intensity of this phase was reduced, illustrating the underlying delithiation process. This can be further demonstrated in the ex situ XPS results by the gradually increase of the deconvoluted
$\mathrm{Co}^{2+}$ peak intensity with discharge proceeding (Fig. 7). Other deconvoluted XPS results ( $\mathrm{C} 1 \mathrm{~s}, \mathrm{~N}$ 1s, and Ga 2p) exhibit an absence of obvious changes (Fig. 7). This is the first example of Li-ion storage in a collapsed PBAs via bonding with one of the host species.

The electrochemical impedance spectra of GaHCCo is shown in Fig. 6c, with a depressed semicircle (charge transfer process) connected with an oblique line (mass transfer process). The material exhibits a charge transfer impedance of $c a .200 \Omega$. In a controlled experiment, we also measure the capacity of the
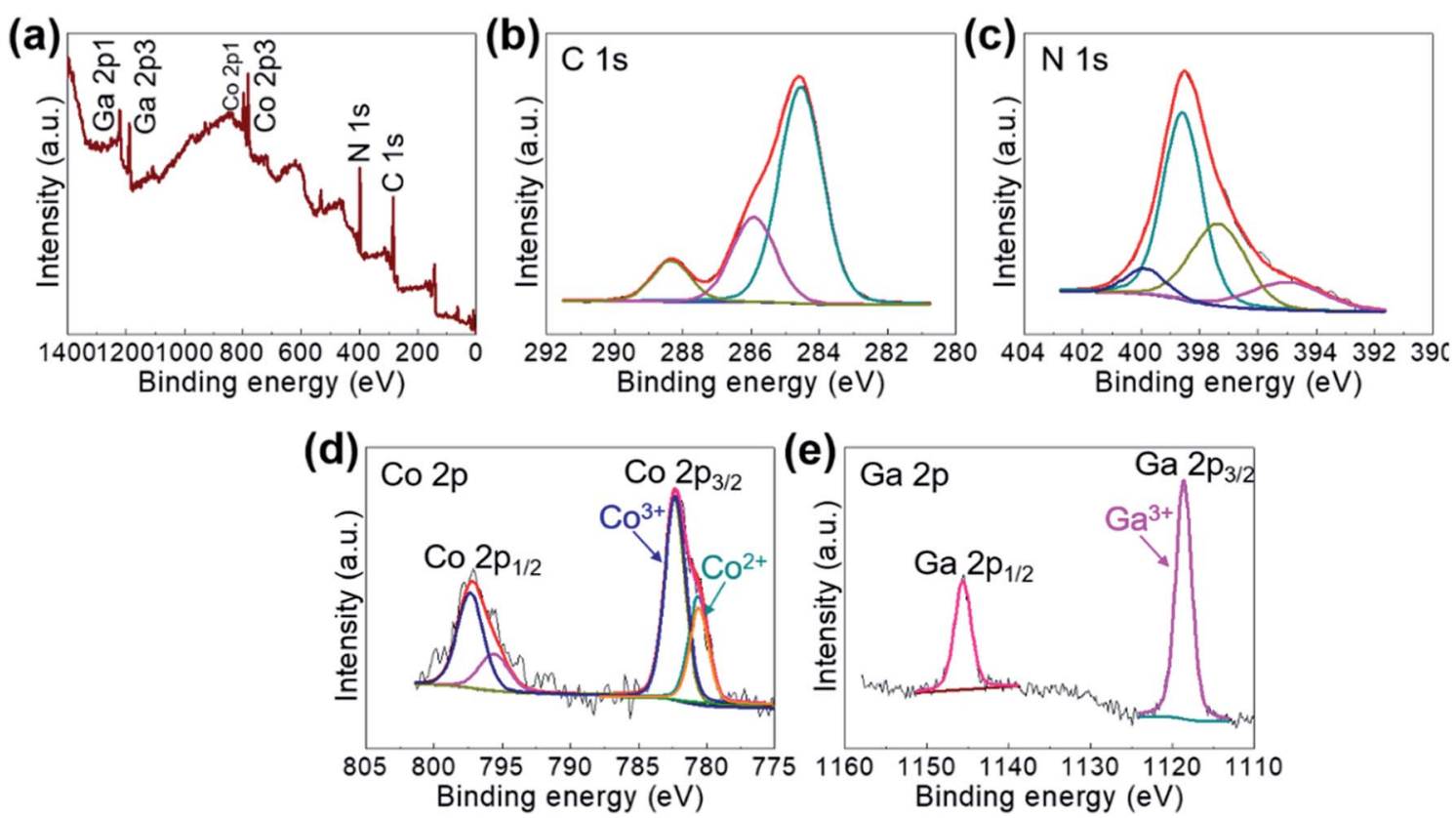

Fig. 3 (a) Wide-survey and deconvoluted (b) C 1s, (c) N 1s, (d) Co 2p, and (e) Ga 2p XPS spectra of the GaHCCo. 


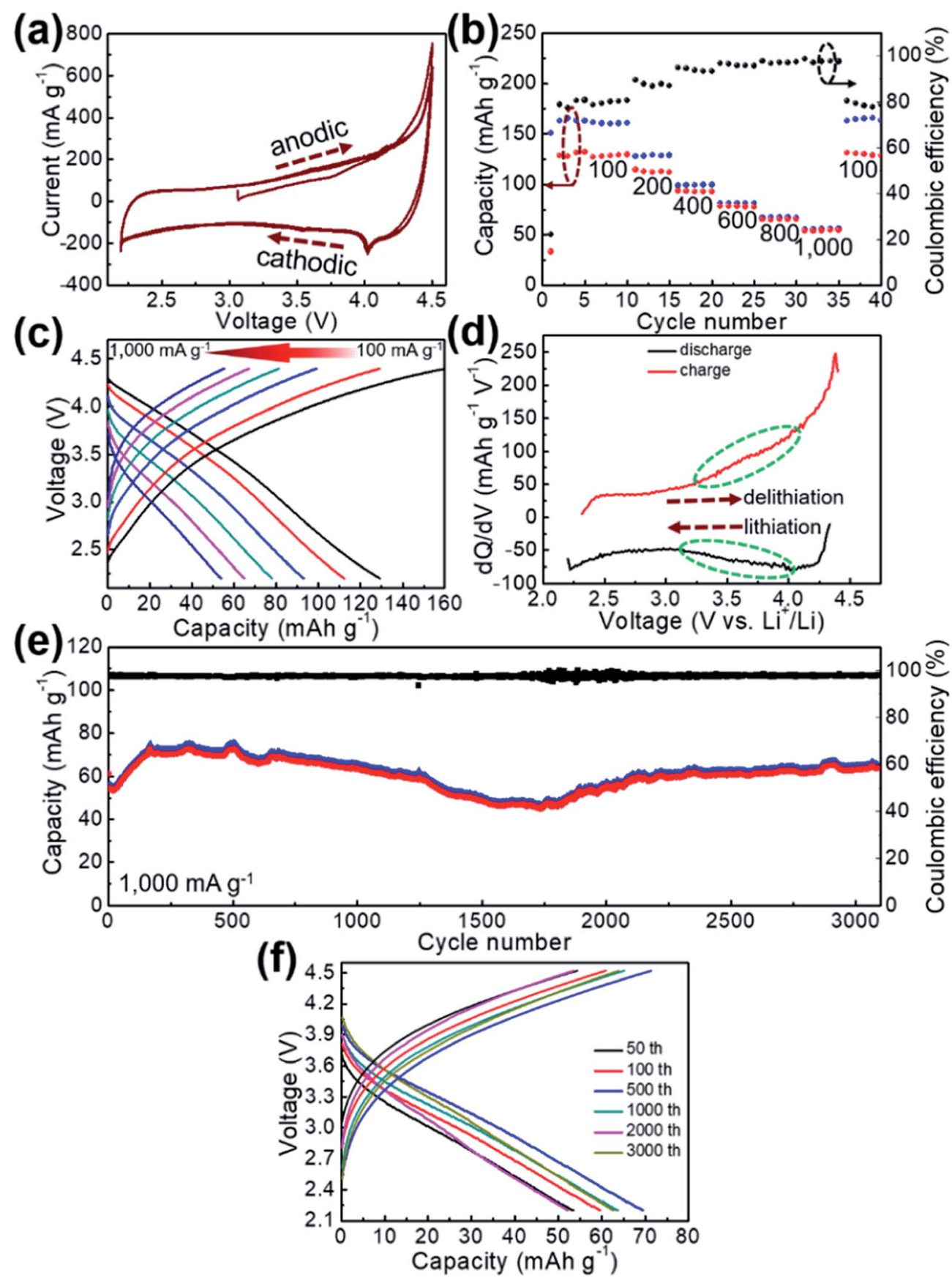

Fig. 4 (a) CV curves, (b) rate performance, (c) corresponding voltage profiles, (d) dQ/dV curves, (e) long-term repeated cycling test, and (f) representative voltage profiles of the GaHCCo.

bare graphite paper current collector, which shows a negligible capacity (Fig. 6d).

To analyze the contributions of diffusion-controlled and capacitive Li-ion storage contributions to the total Li-ion storage capacity of GaHCCo qualitatively and quantitatively, we carried out CV measurements for the GaHCCo cathode at different scan rates $\left(0.5-2.5 \mathrm{mV} \mathrm{s}^{-1}\right)$ within the same potential window of 2.2$4.5 \mathrm{~V} v s$. $\mathrm{Li}^{+} / \mathrm{Li}$ (Fig. 8a). The curves obtained at all scan rates displayed a quasi-rectangular shape. To investigate this capacitive feature further, the dependence of response current $(i)$ on scan rate $(v)$ is modeled using the following power law: $i=a v^{b}$.
The fitting of a $\log (i) v s . \log (v)$ plot is expected to afford a straight line with slope $b ; b=0.5$ corresponds to diffusioncontrolled Li-ion storage, indicating a faradaic electrochemical reaction, whereas $b=1.0$ corresponds to capacitive Liion storage. Here, $b$-values between 0.5 and 1.0 are observed, which indicates the concomitant nature of diffusion-controlled and capacitive Li-ion storage (Fig. 8b). The relative contributions of these storage mechanisms are further calculated by reexpressing eqn (1) as:

$$
i=k_{1} v+k_{2} v^{1 / 2}
$$



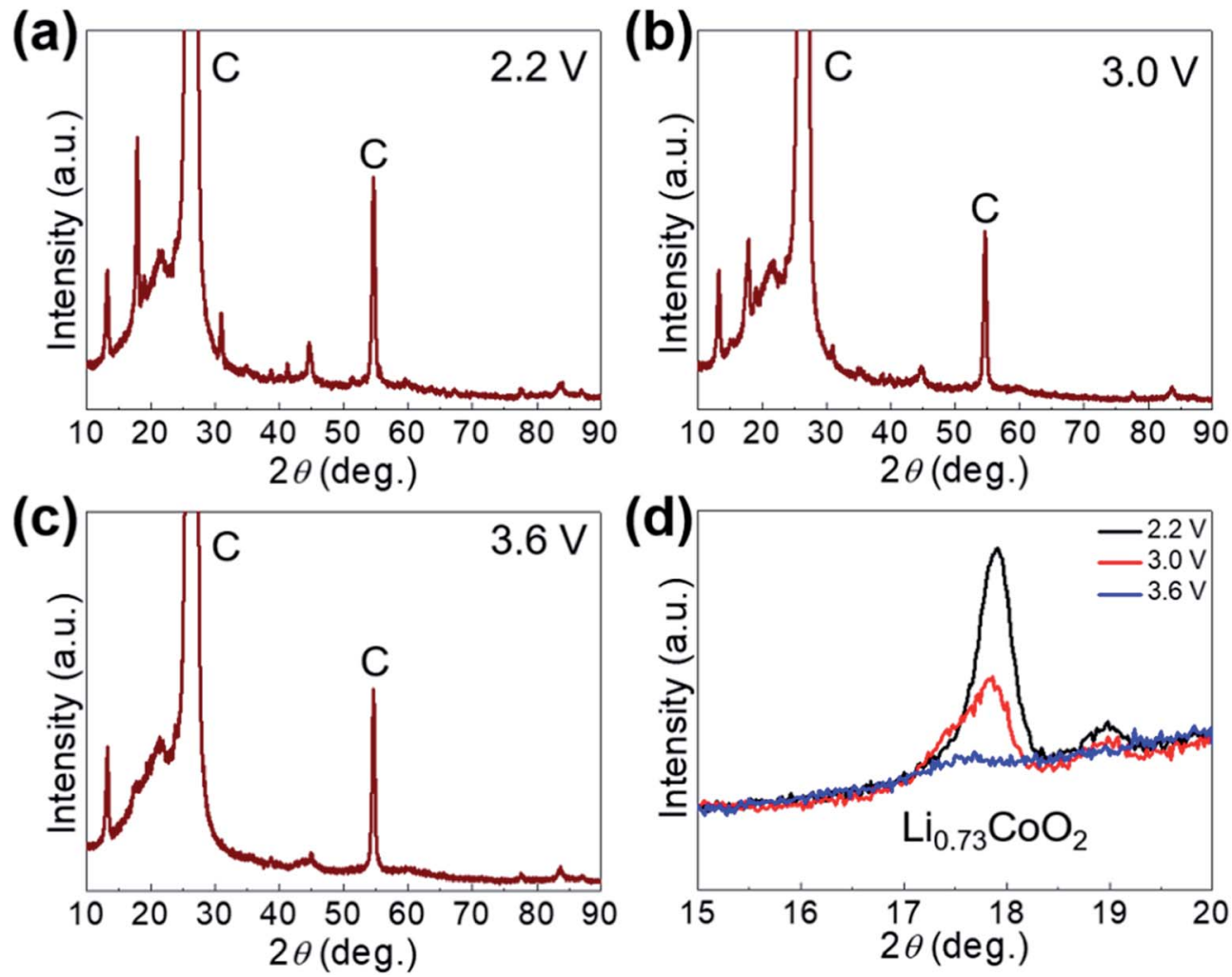

Fig. 5 (a) Ex situ XRD patterns of GaHCCo at (a) $2.2 \mathrm{~V}$, (b) $3.0 \mathrm{~V}$, (c) $3.6 \mathrm{~V}$, and (d) magnified XRD peaks.

where $k_{1} v$ represents the contribution of the $b=1$ case, and $k_{2} v^{1 / 2}$ represents the contribution of the $b=0.5$ case. The obtained results are presented in Fig. 8c, which shows that at
$0.5 \mathrm{mV} \mathrm{s}^{-1}, 30 \%$ of the total capacity corresponded to capacitive $\mathrm{Li}^{+}$storage. This contribution increases with increasing scan rates $\left(49 \%\right.$ at $\left.2.5 \mathrm{mV} \mathrm{s}^{-1}\right)$, which indicates
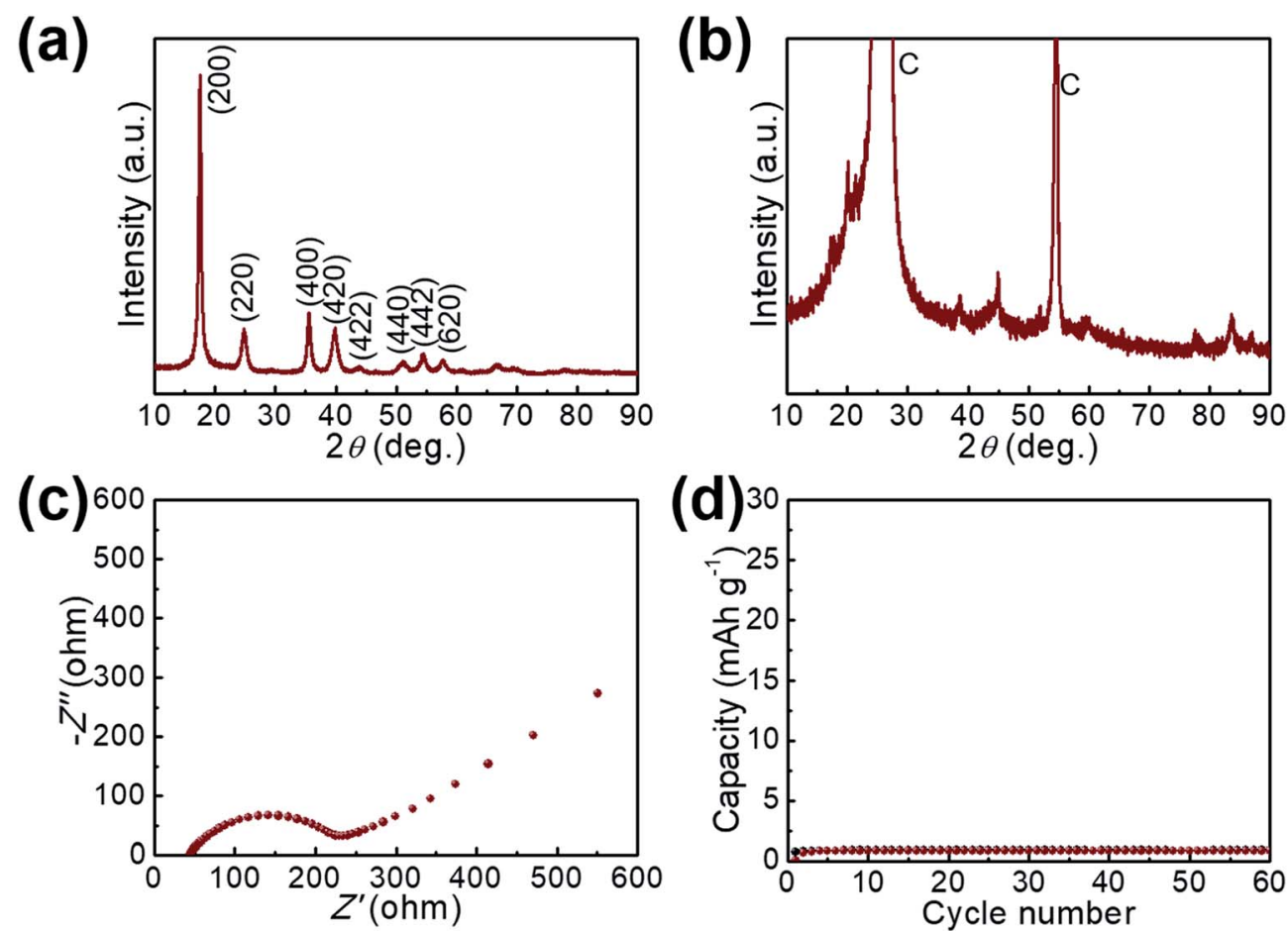

Fig. 6 XRD pattern of the GaHCCo (a) before and (b) after cycling test. (c) EIS spectrum of the GaHCCo. (d) The charge/discharge cycling measurement of the bare graphite paper current collector. 

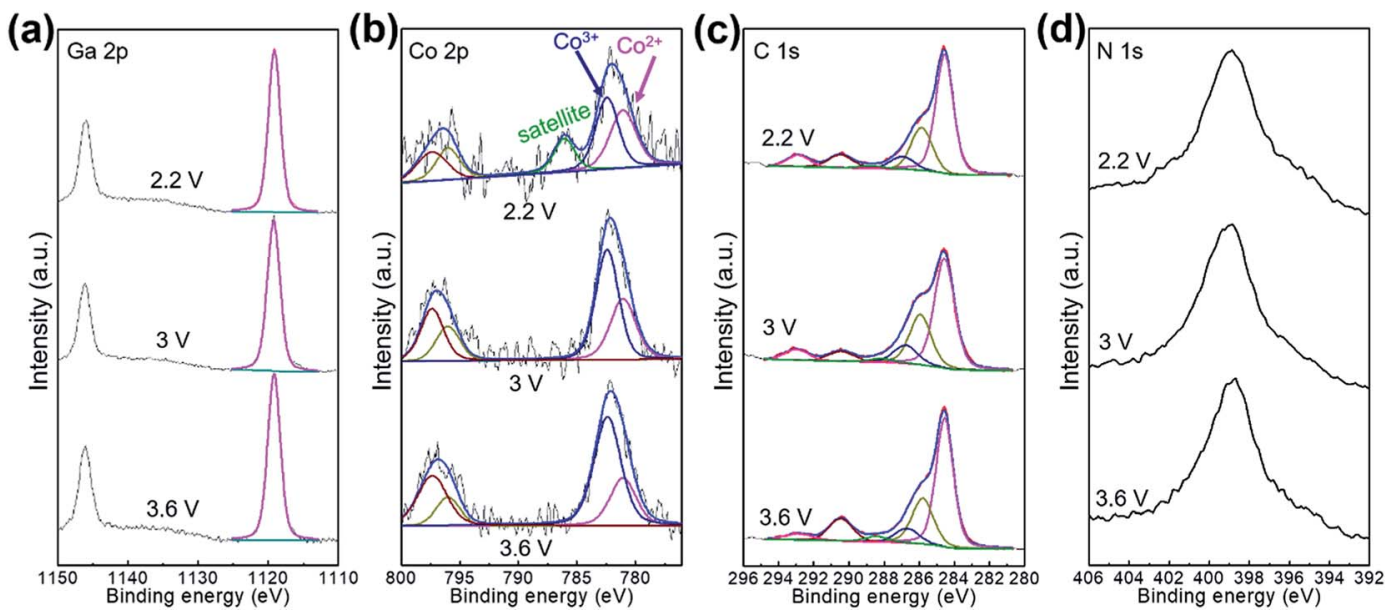

Fig. 7 Ex situ XPS characterizations for the electrode charged at 2.2, 3.0, and 3.6 V.
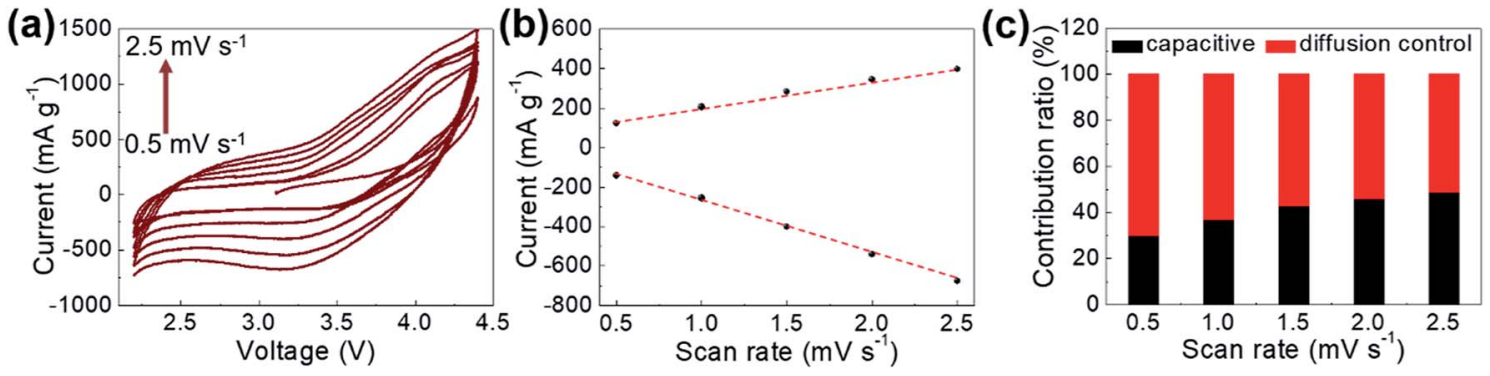

Fig. 8 (a) CV curves obtained at various scan rates, (b) response currents at $3.1 \mathrm{~V}$ when scanned at different rates, and (c) contribution ratios of capacitive and diffusion-controlled Li-ion storage.

that the faradaic redox reaction dominated the Li-ion storage process.

\section{Conclusions}

In this study, we conduct the formation of GaHCCo with an Ncoordinated trivalent metal ion $\left(\mathrm{Ga}^{3+}\right)$ as a cathode material for LIBs. The PBA depicts a face-centered cubic structure with a unique thermally sensitive property. In addition, the synthesized GaHCCo exhibits long-term charge/discharge stability, having a capacity retention of $75 \%$ after 3000 cycles of repeated cycling test with an extremely high coulombic efficiency of $98 \%$, benefiting from a solid-state diffusion-controlled Li-ion storage process. In particular, the Li-ion storage in the GaHCCo is mainly attributed to the Co species by forming a Li/Co compound. This work will pave the way toward the study of PBAs constructed with other trivalent metal ions coordinated to $\mathrm{N}$ end of cyanides and provides more insights into the development of high-performing LIBs in the future.

\section{Conflicts of interest}

There are no conflicts to declare.

\section{Acknowledgements}

This research was supported by Korea Institute of Science and Technology Future Resource Program (2E29400). Furthermore, the financial supports of the Future Material Discovery Program (2016M3D1A1027666), the Basic Science Research Program (2017R1A2B3009135) through the National Research Foundation of Korea are appreciated, and China Scholarship Council (201808260042).

\section{References}

$1 \mathrm{~K}$. Zhang, R. S. Varma, H. W. Jang, J.-W. Choi and M. Shokouhimehr, J. Alloys Compd., 2019, 791, 911-917.

2 W. Li, Y. Yang, G. Zhang and Y.-W. Zhang, Nano Lett., 2015, 15, 1691-1697.

3 S.-H. Yu, M. Shokouhimehr, T. Hyeon and Y.-E. Sung, ECS Electrochem. Lett., 2013, 2, A39-A41.

4 M. Shokouhimehr, S. H. Yu, D. C. Lee, D. Ling, T. Hyeon and Y. E. Sung, Nanosci. Nanotechnol. Lett., 2013, 5, 770-774.

5 Q. Xu, J.-Y. Li, J.-K. Sun, Y.-X. Yin, L.-J. Wan and Y.-G. Guo, Adv. Energy Mater., 2017, 7, 1601481.

6 J. Zhou, J. Qin, X. Zhang, C. Shi, E. Liu, J. Li, N. Zhao and C. He, ACS Nano, 2015, 9, 3837-3848. 
7 S.-H. Yu, X. Guo, D. Ling, D. Y. Chung, A. Jin, M. Shokouhimehr, T. Hyeon and Y.-E. Sung, RSC Adv., 2014, 4, 37365-37370.

8 S.-H. Yu, M. Park, H. S. Kim, A. Jin, M. Shokouhimehr, T.-Y. Ahn, Y.-W. Kim, T. Hyeon and Y.-E. Sung, RSC Adv., 2014, 4, 12087-12093.

9 Y. Sun, N. Liu and Y. Cui, Nat. Energy, 2016, 1, 16071.

10 K. Chen, Z. Sun, R. Fang, Y. Shi, H.-M. Cheng and F. Li, Adv. Funct. Mater., 2018, 28, 1707592.

11 Q. Pang, X. Liang, C. Y. Kwok and L. F. Nazar, Nat. Energy, 2016, 1, 16132.

12 Y.-B. Huang, J. Liang, X.-S. Wang and R. Cao, Chem. Soc. Rev., 2017, 46, 126-157.

13 W.-T. Koo, S.-J. Choi, S.-J. Kim, J.-S. Jang, H. L. Tuller and I.-D. Kim, J. Am. Chem. Soc., 2016, 138, 13431-13437.

14 Z. Wang, R. Tan, H. Wang, L. Yang, J. Hu, H. Chen and F. Pan, Adv. Mater., 2018, 30, 1704436.

15 Y. You, X. Yu, Y. Yin, K.-W. Nam and Y.-G. Guo, Nano Res., 2015, 8, 117-128.

16 Y. You, X. L. Wu, Y. X. Yin and Y.-G. Guo, Energy Environ. Sci., 2014, 7, 1643-1647.

17 T. Wei, M. Zhang, P. Wu, Y.-J. Tang, S.-L. Li, F.-C. Shen, X.-L. Wang, X.-P. Zhou and Y.-Q. Lan, Nano Energy, 2017, 34, 205-214.
18 C. Guan, X. Liu, W. Ren, X. Li, C. Cheng and J. Wang, Adv. Energy Mater., 2017, 7, 1602391.

19 M. Shokouhimehr, E. S. Soehnlen, J. Hao, M. Griswold, C. Flask, X. Fan, J. P. Basilion, S. Basu and S. D. Huang, J. Mater. Chem., 2010, 20, 5251-5259.

20 X. Hou, G. Zhu, X. Niu, Z. Dai, Z. Yin, Q. Dong, Y. Zhang and X. Dong, J. Alloys Compd., 2017, 729, 518-525.

21 J. Shao, J. Feng, H. Zhou and A. Yuan, Appl. Surf. Sci., 2019, 471, 745-752.

22 K. Zhang, T. H. Lee, H. W. Jang, M. Shokouhimehr and J.-W. Choi, Electron. Mater. Lett., 2019, 1-10.

23 F. J. Luque, I. A. Kowalik, J. P. P. Ruiz, M. Á. Niño, H. PrimaGarcía, F. M. Romero, D. Arvanitis, E. Coronado, R. Miranda and J. J. Miguel, J. Mater. Chem. C, 2018, 6, 8171-8186.

24 P. Bhatt, S. S. Meena, M. D. Mukadam, B. P. Mandal, A. K. Chauhan and S. M. Yusuf, New J. Chem., 2018, 42, 4567-4578.

25 Q. Wang, S. He, N. Wang, J. Zhao, J. Fang and W. Shen, New J. Chem., 2016, 40, 3244-3251.

26 B. Mazinani, M. Kazazi, G. Mobarhan and M. Shokouhimehr, JOM, 2019, 71, 1499-1506.

27 R. A. Huggins, J. Power Sources, 1999, 81, 13-19.

28 L. Deng, Z. Yang, L. Tan, L. Zeng, Y. Zhu and L. Guo, Adv. Mater., 2018, 30, 1802510. 